\title{
Numerical analysis of an entire ceramic kiln under actual operating conditions for the energy efficiency improvement
}

\author{
Massimo Milani*, Luca Montorsi, Matteo Stefani \\ Department of Sciences and Methods for Engineering \\ University of Modena and Reggio Emilia, Reggio Emilia, Italy \\ e-mail: massimo.milani@unimore.it \\ Roberto Saponelli, Maurizio Lizzano \\ SACMI SC
}

\begin{abstract}
The paper focuses on the analysis of an industrial ceramic kiln in order to improve the energy efficiency and thus the fuel consumption as well as the pollutant emissions. A lumped and distributed parameter model of the entire system is constructed to simulate the performance of the kiln under actual operating conditions. The model is able to predict accurately the temperature distribution along the different modules of the kiln and the operation of the many natural gas burners employed to provide the required thermal power. Furthermore, the temperature of the tiles is also simulated so that the quality of the final product can be addressed by the modelling. Numerical results are validated against experimental measurements carried out on a real ceramic kiln during regular production operations.

The developed numerical model demonstrates to be an efficient tool for the investigation of different design solutions for the kiln's components. In addition, a number of control strategies for the system working conditions can be simulated and compared in order to define the best trade off in terms of fuel consumption, emissions and product quality. In particular, the paper analyzes the effect of a new burner type characterized by internal heat recovery capability aimed at improving the energy efficiency of the ceramic kiln. The fuel saving and the relating reduction of carbon dioxide emissions resulted in the order of $10 \%$ when compared to the standard burner.
\end{abstract}

\section{KEYWORDS}

Lumped and distributed parameter model, heat transfer, energy efficiency, emissions

\footnotetext{
* Corresponding author
} 


\section{INTRODUCTION}

The ceramic industry is well known to be characterized by energy intense processes. Main process where a large amount of energy is employed is the kiln for the firing of the tiles. Recent regulations for the energy consumption require a more accurate design of the kiln in order to limit the fuel or electricity use [1]. Furthermore, the environmental concerns drive the design towards cleaner systems and more stringent limits about the pollutant emissions [2,3].

Different approaches have been adopted for the optimization of the kilns' performance. In particular, a theoretical formulation for the prediction of the kiln operating characteristics has been proposed by [4] under regime conditions and many simplifications to the physical phenomena had to be made in order to close the mathematical model. Similar approach is adopted also in [5] in which the main focus was the assessment of the heat transfer between the hot air flow and the kiln walls. The numerical simulation becomes a valuable tool in investigating the performance of complex physical systems including different physical phenomena. In [6] a numerical model has been proposed and implemented into a Fortran routine for evaluating the temperature distribution within the walls, gas and tiles along the kiln under steady state assumption.

Indeed, the processes typical for a ceramic kiln are strongly time dependent and in particular the assessment of different control strategies involves the analysis of the transient phase. Lumped and distributed numerical analysis has been extensively adopted for the simulation of complex systems under time dependent operating conditions. In [7, 8] a novel concept for the hydrogen production has been investigated by modelling the entire proposed plant and different operations have been compared in order to define the best energy efficiency configuration. The modelling approach has been also employed for the validation of integrated waste-to-energy systems with low environmental impact and the advantages compared to traditional technologies have been outlined [9]. Despite the dimensional approximation, the 0D/1D models proved also to be able to account for complex physics involving heat transfer phenomena while including the layout of the full system [10].

In this paper, the simulation of a whole industrial ceramic kiln has been carried out by means of a lumped and distributed parameter approach. The performance of the kiln was evaluated under actual operating conditions in order to improve the energy efficiency and thus the fuel consumption as well as the pollutant emissions. Particular care was devoted in modelling the many burners employed in the real kiln and the heat transfer processes between the different elements that encompass the system and effect the final temperature of the tiles and therefore the quality of the product. The results of the numerical model are validated against experimental measurements carried out on a real ceramic kiln during regular production operations. The agreement between the calculations and the measured values demonstrated to be very satisfactory; hence, the lumped and distributed numerical model can be employed for evaluating different kiln configurations and alternatives.

In particular, the effects of a new burner type characterized by internal heat recovery capability has been investigated and the improvement in terms of energy efficiency of the ceramic kiln addressed.

The fuel saving and the relating reduction of carbon dioxide emissions resulted in the order of $10 \%$ when compared to the standard burner. 


\section{ANALYZED CERAMIC KILN}

The ceramic kiln simulated in this paper is real production facility located in the ceramic district in Emilia Romagna - Italy. It is characterized by a production rate of approximately $5,000 \mathrm{~kg} / \mathrm{h}$ of tiles and it is designed for a continuous working load of about 8,700 hours per year.

The entire ceramic kiln includes 43 modules with a length of $2.1 \mathrm{~m}$ each and it can be subdivided into the following 5 sections: pre-heating, firing, fast cooling, slow-cooling and outlet section, see Figure 1.

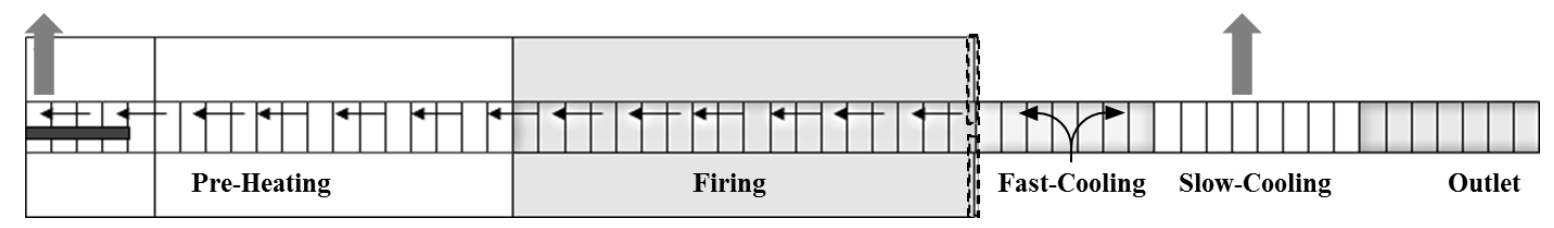

Figure 1. Schematic of the entire ceramic kiln

This study focuses mainly on the first three sections since they demonstrated to be the critical ones for the quality of the tiles and the energy consumption. In particular, an accurate temperature control is mandatory for achieving the desired product quality and avoiding material defects.

In the pre-heating section, no burners are employed and the heating is due to the hot air flow from the burners zone. As far as the energy consumption is concerned, the most important zone is the firing where the burners are installed. The burners are fuelled by natural gas and the fuel rate is controlled for each group of 8 burners as depicted in Figure 2. One group of burners belongs to two modules and there are two groups of burners for each module, one above the roller plane and the second below respectively. The different control of the burners above and below the rollers is critical in achieving different temperatures in these chambers.

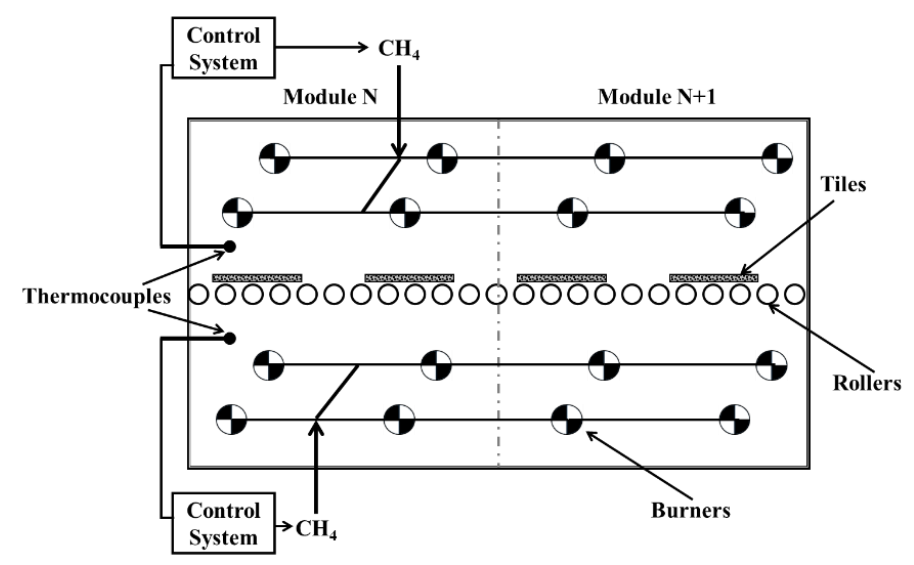

Figure 2. Burners layout in the firing zone

At the end of the firing section, a fireproof wall is positioned in order to separate the cooling zone to the firing zone; main task of this wall is to partition the flow between the firing and fast-cooling sections. Its height is adjusted in order to always direct the flow from the cooling to the firing section and regulate the temperature at the interface of these two regions.

An important characteristic of the analyzed kiln is the opposite direction of the hot air flow and the tile motion. A mentioned before, the air is inducted from the cooling zone by means of a fan located at the beginning of the kiln before the flue gas stack. Therefore, the pressure in the cooling zone is higher than the one in the firing section, which is usually characterized by values 
below the atmospheric pressure. As a consequence, the leakages in the walls of the modules allow the cold ambient air to enter the kiln chambers influencing the temperature of the hot air flow and thus the energy consumption of the entire system. The remaining portion of air in the fast-cooling section exits the kiln through the slow-cooling section and the final outlet stack. An important feature of the analyzed kiln is the counter-current heat transfer mode.

\section{NUMERICAL MODEL}

The lumped and distributed parameters model of the ceramic kiln described in the previous sections is constructed using the LMS Imagine.LAB. AMESim® ${ }^{\circledR}$ code [11]. In particular, the thermo-pneumatic library is used. This library enables to use as a fluid the numerical model of the thermo-dynamic properties of the considered gas and the semi-perfect gases approach is employed. In the simulation the properties of a mixture including three gases (i.e. the exhaust gases from the air/methane combustion) are considered, under the assumption that in every volume the mixture is homogeneous. In addition, the thermal library is used for the simulation of the solid parts of the kiln and their influence on the whole heat exchange is taken into account. The numerical approach employed to study the gas dynamic behavior of the ceramic kiln accounts for the following assumptions:

- the gas efflux through an orifice is mainly $1 \mathrm{D}$, the gas efflux is subsonic, and the influence of the most relevant $2 \mathrm{D}$ and $3 \mathrm{D}$ effects is accounted by introducing proper corrective parameters; - all orifices present a constant (and known) maximum metering area, and they have an infinitely short axial length;

- the gas physical, mechanical and thermodynamic characteristics (density, viscosity, pressure, velocity, temperature...) can be described by their mean values (in space and time);

- the gas physical properties variations with pressure and temperature can be described following a "semi-perfect" approach [12, 13].

The simplifying hypotheses previously mentioned enables to calculate the mass flow-rate as:

$$
\dot{m}=A \cdot C_{q} \cdot C_{m} \cdot \frac{p_{0}}{\sqrt{T_{0}}}
$$

Equation 1, which is a function of inlet absolute pressure and temperature only $\left(\mathrm{p}_{0}, \mathrm{~T}_{0}\right)$, presents two corrective parameters. The first one, $C_{q}$, is the efflux coefficient, and it is generally addressed to modify the reference area used for calculating the fluid velocity. For the purpose of this work, and according to the sensitivity analysis detailed in [14], all the orifices are characterized by adopting an efflux coefficient equal to 0.95 . The second corrective parameter, $C_{m}$, is generally indicated as the mass flow-rate coefficient and, with respect to an isentropic subsonic efflux, it has to be expressed as:

$$
C_{m}=\sqrt{\frac{2 \cdot k}{r \cdot(k-1)} \cdot\left[\left(\frac{p_{1}}{p_{0}}\right)^{2 / k}-\left(\frac{p_{1}}{p_{0}}\right)^{k-1 / k}\right]}
$$

In Equation 2, $k$ represents the isentropic expansion exponent, and it equals the gas specific heats ratio, while $r$ is the gas characteristic constant. Thanks to the adoption of the "semiperfect" approach, the gas physical properties variations with pressure and temperature are modelled considering on one hand the perfect gas Equation of State, on the other hand a polynomial correlation to define the specific heat variations with temperature [12].

$c_{p}=c_{p_{0}} \cdot\left[b_{c}+b_{t} \cdot \Delta T+b_{t_{2}} \cdot(\Delta T)^{2}\right]$

This approach, forcing a physical properties dependency on temperature stronger than on pressure, is valid only for low-medium pressure applications, for which the instantaneous temperature variations are more affecting the thermodynamic variables than the instantaneous pressure variations. 
In the following, the exhaust gas mixture is considered as fluid medium, and the specific heat adopted in Equation 3 is chosen coherently with the specific heat variations with temperature.

\section{Numerical model of the main subsystems}

Figure 3 shows the numerical model of the reference ceramic kiln. The Figure outlines the main parts of the system as shown in the functional layout, see Figure 1. In addition to the thermopneumatic and thermal libraries, the signal library is also adopted to control the fan, the throttle valves and the burners. This library is as well used to set several boundary conditions, such as the external temperature and pressure in the ducts' model, the equivalence ratio for the burners, the initial temperature of the walls and the kiln chambers.

In the following, the numerical models constructed for the main components of the analyzed system are described more in detail.
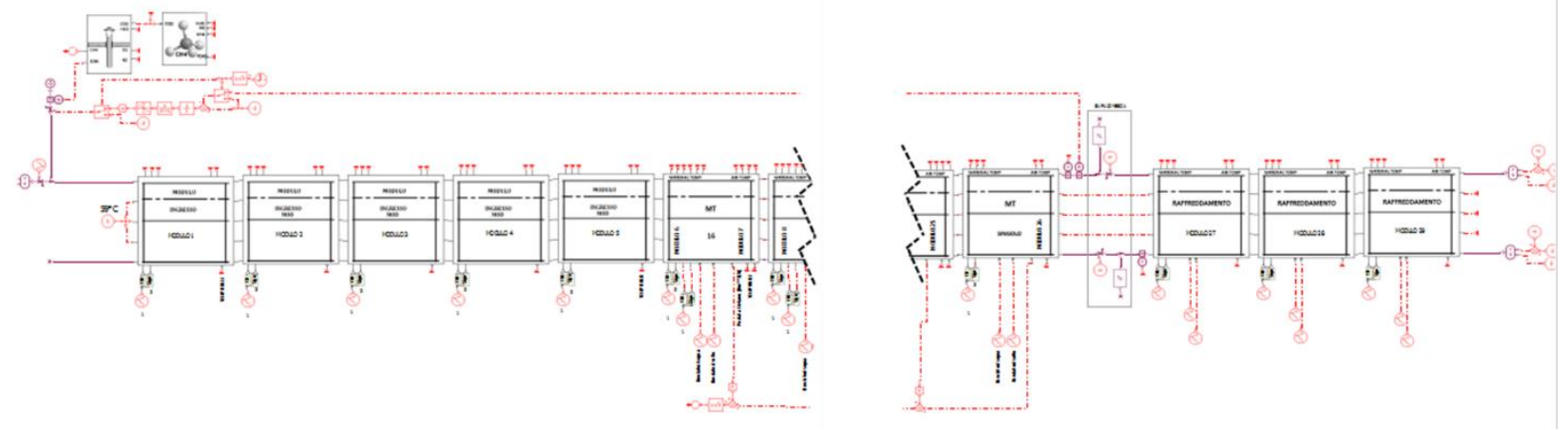

Figure 3 Layout of the numerical model of the reference ceramic kiln

$\underline{\text { Kiln chambers }}$ Particular care is devoted in the modelling of the heat transfer between the kiln's walls and the hot air flow as well as the tiles and the rollers. Conduction, convection and radiation are all taken into account and proper formulations are adopted for the different elements of the kiln. Figure 4 shows a schematic of the heat transfer phenomena included in the simulation and remarks the mutual exchange between the kiln's components that are accounted for in the numerical layout.

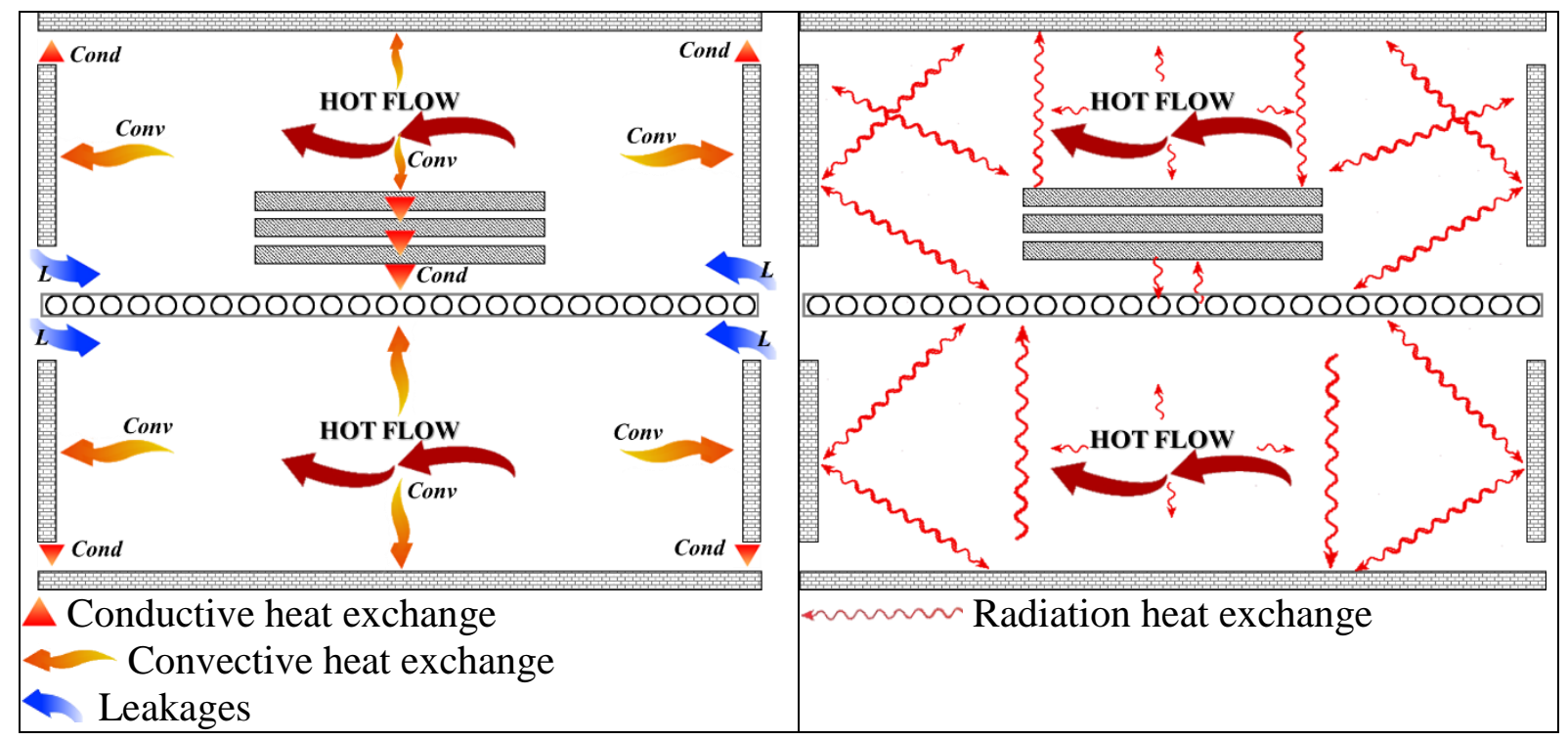

Figure 4. Schematic of the heat transfer among the different components of the kiln module: a) conductive and convective and $b$ ) radiation heat exchange 
The conduction is calculated by means of the following linear formulation:

$d h_{\text {cond }}=\frac{\lambda \cdot A_{\text {cond }}}{t h} \cdot\left(T_{2}-T_{1}\right)$

where $\lambda$ is the thermal conductivity of the material in which the heat flow rate is propagating, $A_{\text {cond }}$ is the exchange area, and th is the distance between the two temperature side under consideration.

As far as the convection is concerned, the following correlation is adopted:

$d h_{\text {conv }}=\frac{N u \cdot \lambda}{c l} \cdot A_{\text {conv }} \cdot\left(T_{f}-T_{w}\right)$

where $\frac{N u \cdot \lambda}{c l}$ is the convective exchange coefficient, $A_{\text {conv }}$ is the exchange area, $T_{f}$ is the mixing temperature of the fluid and $T_{w}$ is the wall temperature, $c l$ is the characteristic length of the heat exchange and $N u$ is the Nusselt number.

The mixed convection is calculated by determining the Nusselt number as follows:

$N u=\sqrt[3]{N u_{\text {free }}^{3}+N u_{\text {forced }}^{3}}$

where $N u_{\text {free }}$ and $N u_{\text {forced }}$ are the Nusselt numbers calculated for the free convection and forced convection cases respectively.

$N u_{\text {free }}=\left(0.825+\frac{0.327 \cdot R a^{1 / 6}}{\left(1+\left(\frac{0.492}{\mathrm{Pr}}\right)^{9 / 16}\right)^{8 / 27}}\right)^{2}$

$N u_{\text {forced }}=0.0296 \cdot \operatorname{Re}^{4 / 5} \cdot \operatorname{Pr}^{1 / 3}$

Finally, the radiative heat flow rate contribution is accounted for by using the following expression:

$$
d h_{r a d}=\frac{\sigma\left(T_{j}^{4}-T_{i}^{4}\right)}{\frac{1-\varepsilon_{i}}{\varepsilon_{i} A_{i}}+\frac{1}{A_{i} F_{i j}}+\frac{1-\varepsilon_{j}}{\varepsilon_{j} A_{j}}}
$$

where $\sigma$ is the Stefan-Boltzmann constant $=5.67 \times 10^{-8} \mathrm{~W} / \mathrm{m}^{2} / \mathrm{K}^{4}, \mathrm{~A}_{i, j}$ is the emitting area of body $i, j$, and $F_{i j}$ is the shape factor from which $A_{i j}$ "sees" $A_{j i}$. For every component included into the kiln chambers model the emitting area and corresponding shape factor is carefully selected as a function of the real geometry and the mutual portion of surface influencing the radiation heat exchange and enforcing the following relationship:

$A_{i} \cdot F_{i j}=A_{j} \cdot F_{j i}$

The properties of the material are set for each element of the kiln chambers, and insulation is also taken into account both in terms of specifications and thickness. Furthermore, leakages between the internal volume of the kiln and the ambient are also included in the simulation. Main contribution to the leakages is the clearance between the rollers and the module walls; the area of the clearance is evaluated and considered in the modelling as a pneumatic connection, through which cold air can enter the kiln and decrease the total efficiency of the production process. 
Usually in the real kiln, the clearance is obstructed by means of an insulating material; thus, in the numerical model the presence of the insulating material is accounted for using a low discharge coefficient for the flow through the leakage connection.

Tiles Main contributions to the heating process of the tiles are the convection with the hot flow rate and the burner flame, the conduction through the contact area to the rollers and the radiative exchange with the cambers' walls and the rollers. The thermal power transferred during these processes is evaluated by means of the expressions detailed previously.

In order to calculated the temperature gradient within the tile body, the model of the ceramic product is subdivided into three layers having the same thickness. The first one represents the surface of the tile in contact with the rollers and exchanges heat with them through the conduction and radiation processes. The second layer models the inner part of the tile and exchanges heat only with the lower and upper layers through conduction. Finally, the upper layer simulates the top surface of the tile and is heated manly by the convection heat transfer with the hot air flow and the burner flame as well as by the radiation with the high temperature kiln's walls.

In order to model the tile motion with respect to each module, an ad-hoc procedure is implemented into the simulation code. The residence time of the tile in each section of the kiln is calculated as a function of the tile velocity and module length; thus, the initial temperature of the thermal mass element modelling each layer of the tile is reset to the final temperature of the tile layer from the previous module after the residence time is elapsed. Therefore, the effects of new ceramic material entering each module are accounted for, and the minimum and maximum temperature values reached by the tiles in every section of the kiln are predicted.

$\underline{\text { Burners }}$ The standard natural gas burner type is simulated by means of the output thermal power that the real burner is producing in the considered operating condition. The same control strategy for setting the fuel mass flow rate consumed by each burner is implemented in the numerical model of the kiln. Thus, as a function of the temperature set point for each chamber and the calculated temperature reached in that chamber, the fuel flow to the burners is adjusted in order to meet the desired temperature. Therefore, the natural gas consumption is predicted by the simulation as a function of the thermal balance of the chamber as in the real kiln. Indeed, the burners are not controlled individually, but a group of eight burners is regulated by the same controller, see Figure 2.

Particular attention is devoted to account for the turbulence created by the burner flame, which influences the heat exchange of the tile top surface and the chambers' walls. In fact, due the high velocity of the flame jet, the convective heat transfer is much more efficient in the areas reached directly by the burner gases. Therefore, for the firing zones of the kiln, the convective coefficient is not only calculated as a function of the hot air flow mean velocity, but the burnt gases velocity is also accounted for and the value is derived from experimental measurements of that regions.

\section{VALIDATION OF THE NUMERICAL MODEL}

The prediction capabilities of the numerical model described in the previous section are validated by comparing the calculated results against the experimental measurements carried out on a real ceramic kiln during regular production operations. The tiles produced during the tests have dimensions of $300 \times 300 \mathrm{~mm}$ and height equal to $8 \mathrm{~mm}$ and the kiln is run with a production rate of approximately $5,000 \mathrm{~kg} / \mathrm{h}$. The "firing curve" set by the main the control system determines the set point temperature for each chamber of each module for the current production. The real temperature reached by the different kiln sections is measured with a PT 100 thermocouple in a number of points within the volume, in order to have a distribution of 
the values representing the considered chamber. The temperature in the measured points results quite uniform and thus a single value is compared to the calculations. Both the chambers above and below the rollers' plane are considered in the measurements, in order to investigate the upper and lower flow of the kiln.

Furthermore, the temperature of the rollers is measured close to the middle of the module width. The experimental values are compared with the numerical calculations in the same section of the kiln layout.

Figures 5 and 6 show the firing curves in the kiln upper and lower chambers and the comparison between the measured and calculated values of the temperature. The data are divided by a reference temperature value in order to have non-dimensional quantity. The numerical results demonstrate to match accurately the experimental measurements and the trend of the temperature distribution of the hot air flow along the kiln length is correctly predicted.

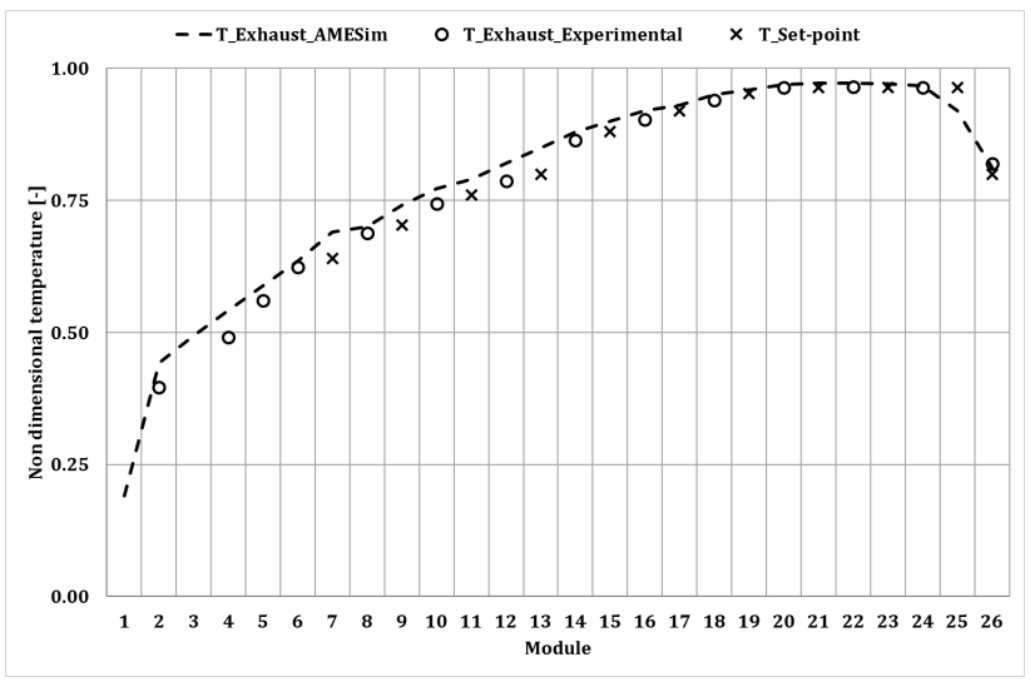

Figure 5. Firing curve and comparison between the measured and calculated values of the temperature in the kiln upper chambers

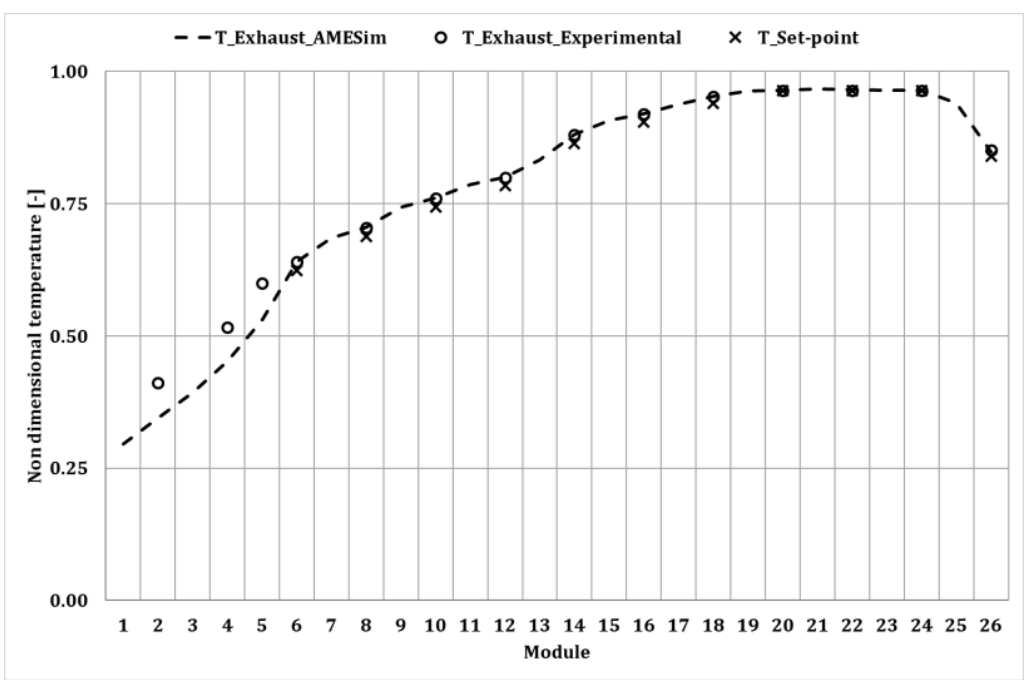

Figure 6. Firing curve and comparison between the measured and calculated values of the temperature in the kiln lower chambers 
Figure 7 shows the measured and calculated non-dimensional temperature of the rollers. It can be noticed that a good agreement between experiments and numerical results can be obtained also for the solid components of the kiln and the trend is captured through all its length.

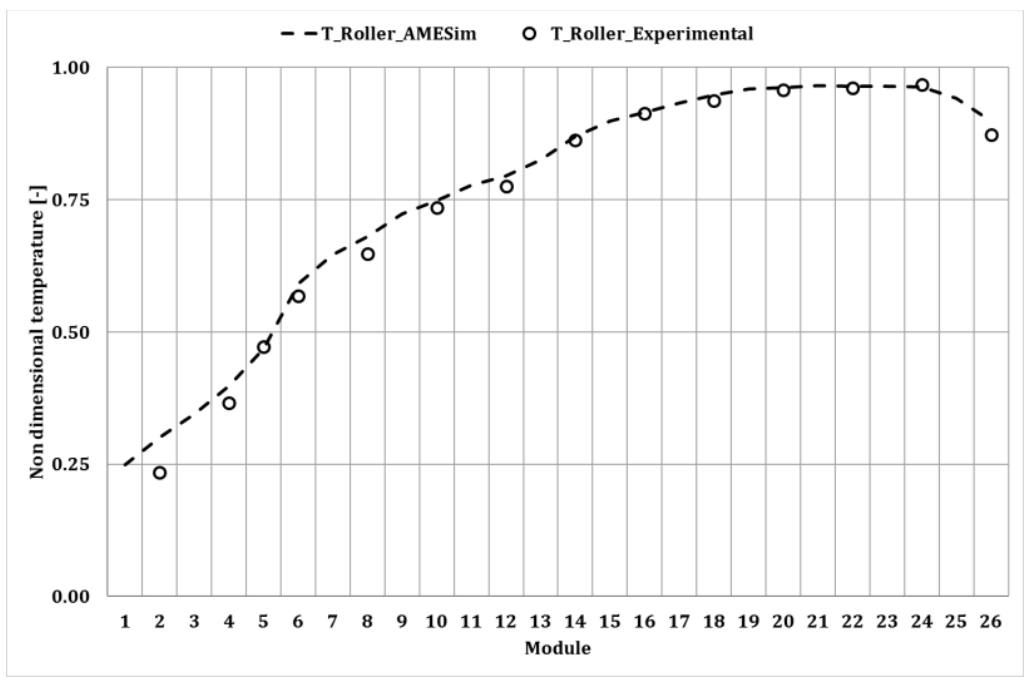

Figure 7. Comparison between the measured and calculated values of the temperature of the kiln rollers

Finally, the real kiln fuel consumption is compared to the predicted values, see Figure 8 . The real kiln methane consumption is taken as a reference and the calculated value is plotted as a ratio to the real amount. The numerical approach demonstrates also in case of the fuel consumption to be able to predict accurately the energy efficiency of the system.

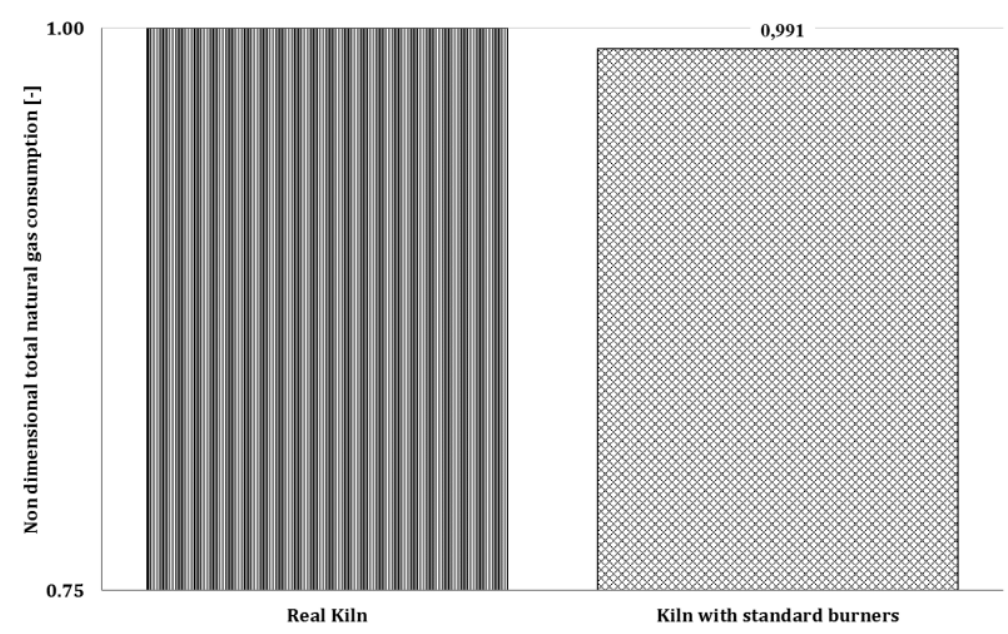

Figure 8. Comparison between the measured and calculated values of the kiln fuel consumption

Once the numerical model is validated against experimental measurements, simulation can be employed to widen the analysis of the kiln operation by investigating quantities that are difficult to be measured. For instance, Figure 9 shows the non-dimensional methane consumption during the production phase for each group of 16 burners that correspond to 2 modules. It can be outlined that burners in the initial and final regions of the kiln are characterized by the larger 
fuel consumption, since they are influenced by the cold zones at the beginning of the kiln and before the fast-cooling section.

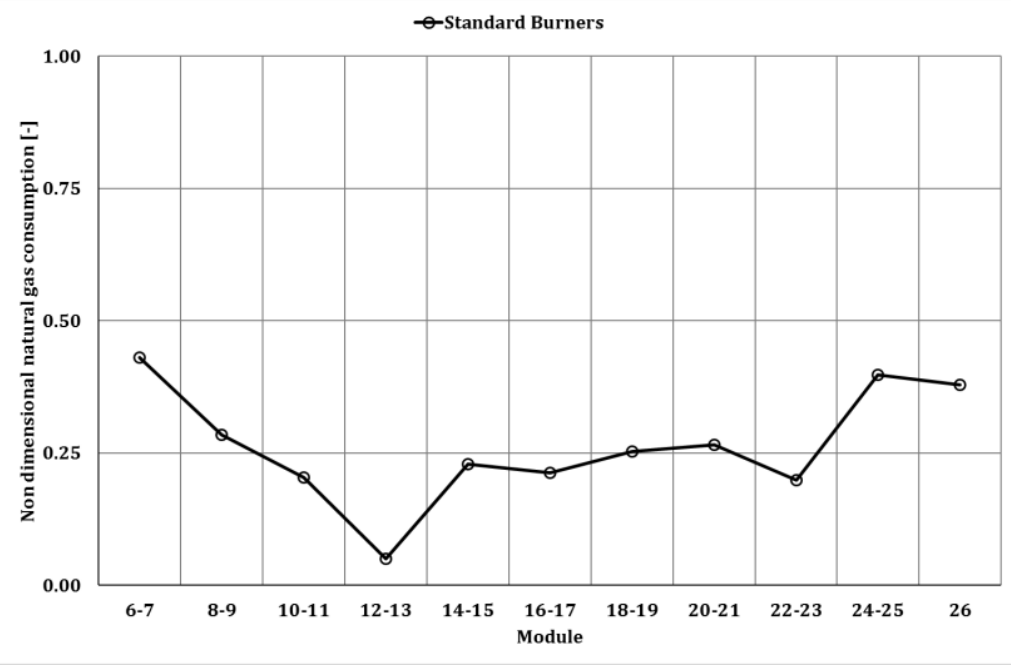

Figure 9. Methane consumption during the production phase for each group of burners

In addition, the numerical model can also evaluate the temperature of the ceramic material and its trend along the kiln length can be compared to the hot air temperature profile, see Figure 10. The non-dimensional temperatures of the three layers of the tile model are very similar, and their difference can be visible in the graph only at the beginning of the kiln, where the surface temperature is significantly larger than the ones for the other two layers. This result can also be explained by the small thickness of the ceramic tile considered in the test (i.e. $8 \mathrm{~mm}$ ). Furthermore, the firing process is tuned in order to keep this temperature difference as low as possible, in order to minimize the residual internal stresses that many form within the tile in case of a remarkable thermal gradient. When comparing the hot air and tile temperatures, it can be noticed that after the entrance in the firing zone, module \# 6 , the tiles reach the air flow temperature quite quickly, and in module \# 10 the ceramic material is at the same temperature as the air.

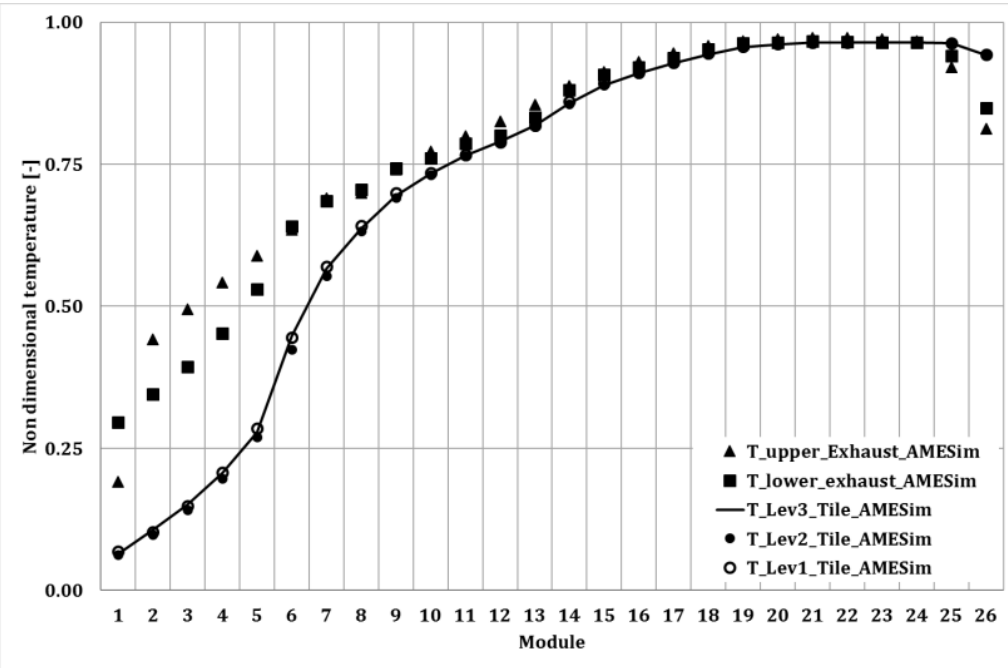

Figure 10. Comparison of the non-dimensional temperature of the tile with the hot air temperature of the upper and lower chamber 


\section{OPTIMIZATION OF THE KILN PERFORMANCE}

The proposed numerical model is employed for investigating the effects on the kiln energy efficiency of a new heat-recovery burner type. The new burner concept exploits the main hot air flow to preheat the oxidizing air used in the burner for the fuel combustion. Figure 11 shows the main functioning of the new burner; the oxidizing air and the fuel flow through two separate channels up to the burner nozzle. The combustion air flows in the outer duct and is heated up by the hot air drawn from the main kiln chamber in the opposite direction. The hot air does not enter again the kiln but exits from a separate piping where a valve regulates the flow rate on the basis of the air temperature at the burner outlet section. Main advantages of the heat-recovery burner are the stability of temperature and pressure in every production conditions, the simplification of the chamber of the ceramic kiln and the optimization of the control of the firing curve. Furthermore, the new burner is expected to reduce to fuel consumption holding constant the firing curve and the product quality.

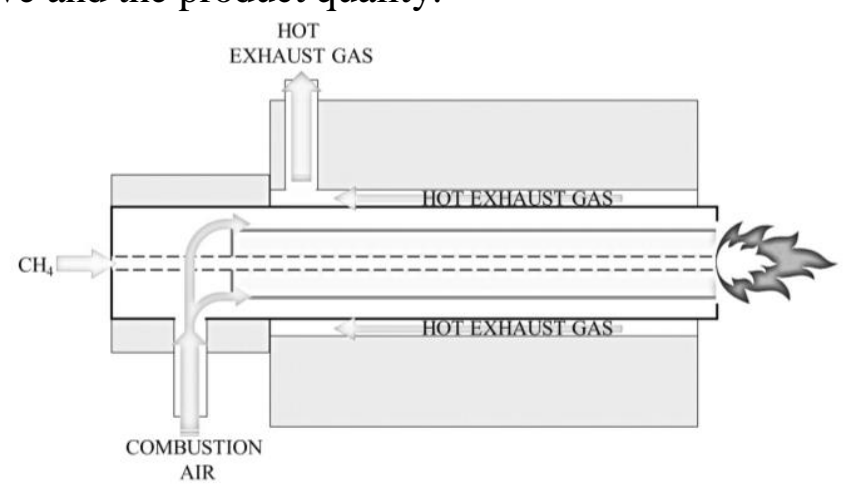

Figure 11. Schematic of the new heat-recovery burner

A detailed lumped and distributed parameter model of the regenerative burned is created using the LMS Imagine Lab. code, see Figure 12. Particular care is devoted in constructing the channels for the flows of the three fluids involved: the methane, the oxidizing air and the hot air from the kiln chamber. The heat transfer process among the considered fluids and the solid parts that constitute the burner pipes and heat recovery system is accounted for using the formulation detailed in the previous section. The effects of the valve regulating the regenerative flow are also accounted for in the modelling, thus the position of the valve that optimizes the operation of the new burner is determined as a result of the numerical approach.

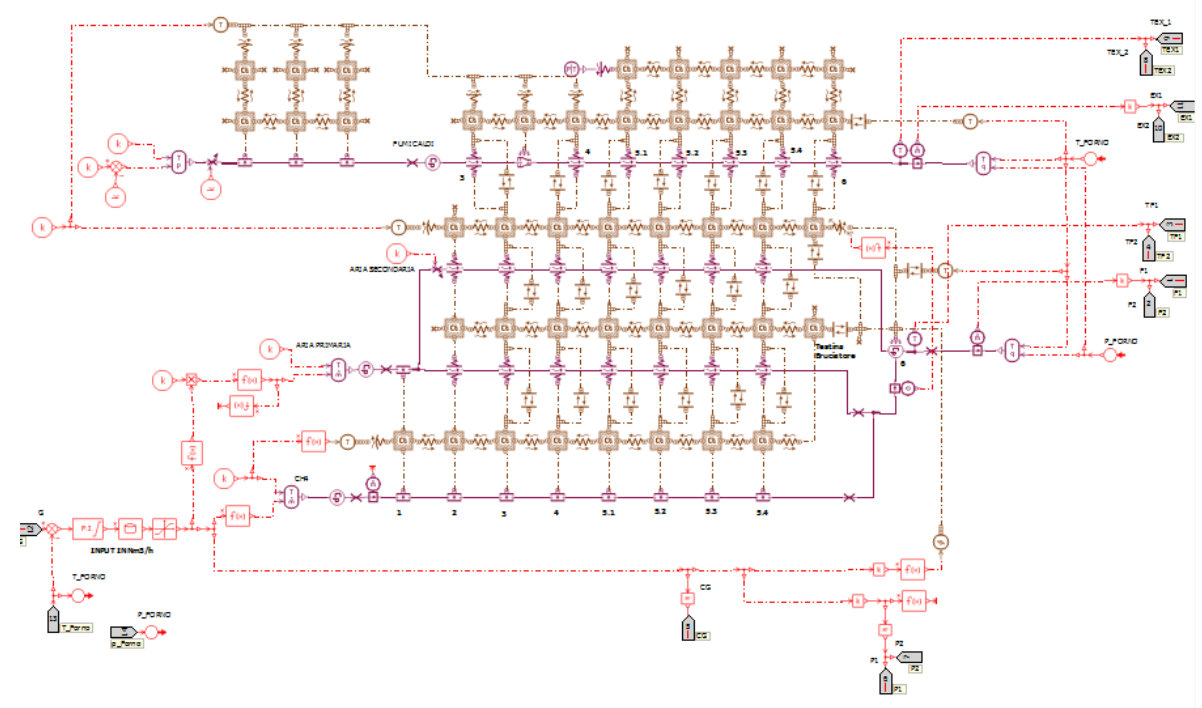

Figure 12. Layout of the numerical model of the new heat-recovery burner 
The 0D/1D model of the regenerative burner is then employed to replace the standard burner used in the numerical layout depicted in Figure 3 in the last 12 modules of the ceramic kiln. Hence, the simulation of the whole kiln equipped with the new burners is calculated under the same operating conditions adopted in the previous analysis and the results of the advanced configuration are addressed. Figures 13 and 14 compare the temperature obtained in the kiln upper and lower chambers using the standard and the heat-recovery burners. It can be noticed that the temperature profiles are very similar, thus the overall behavior of the kiln can be assumed as unvaried. This consideration is confirmed also by the temperature profile of the ceramic tile along the kiln length, see Figure 15. Therefore, it can be concluded that the two analyzed kiln configurations operate in the same way and produce tiles with the same quality; thus, the fuel consumptions can be compared holding constant the output of the kiln.

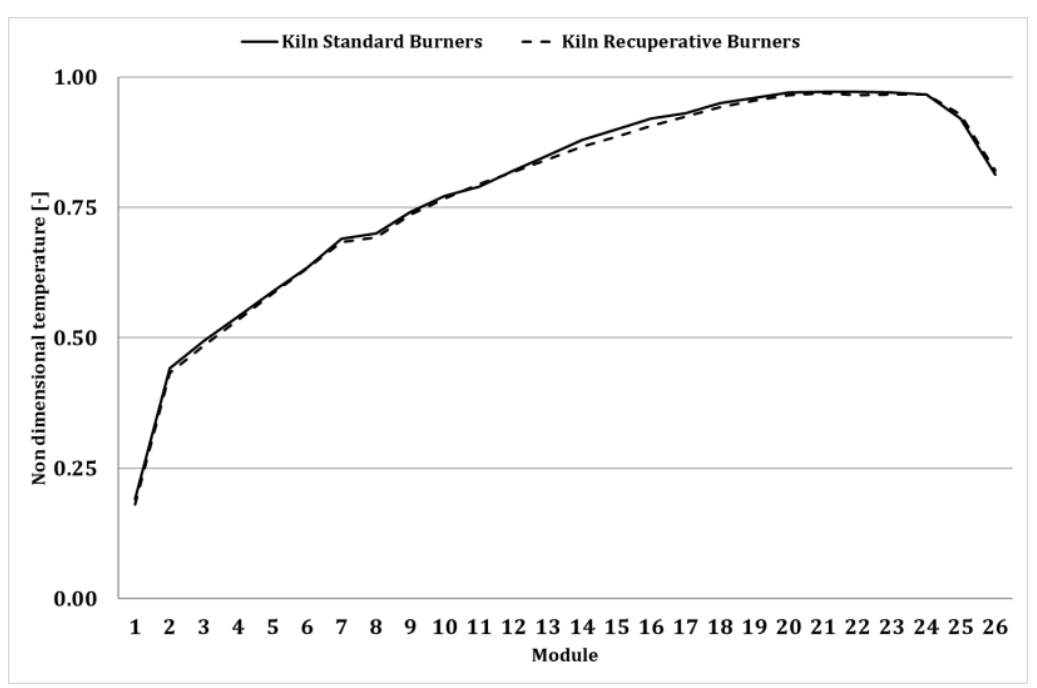

Figure 13. Comparison of the temperature obtained in the kiln upper chambers using the standard and the heat-recovery burner

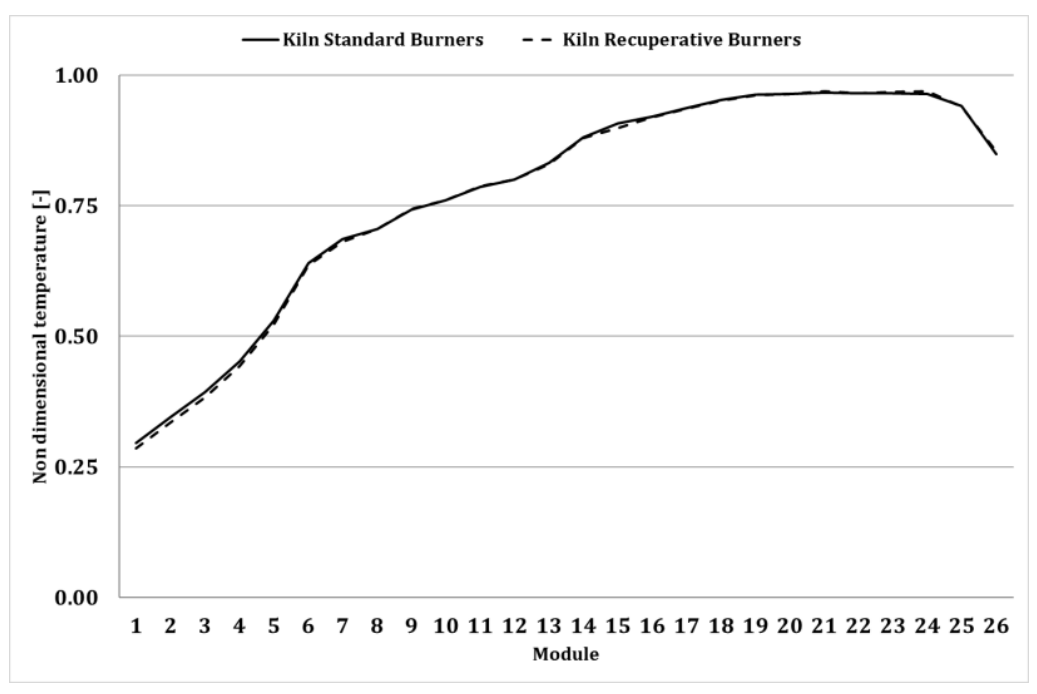

Figure 14. Comparison of the temperature obtained in the kiln lower chambers using the standard and the heat-recovery burner 


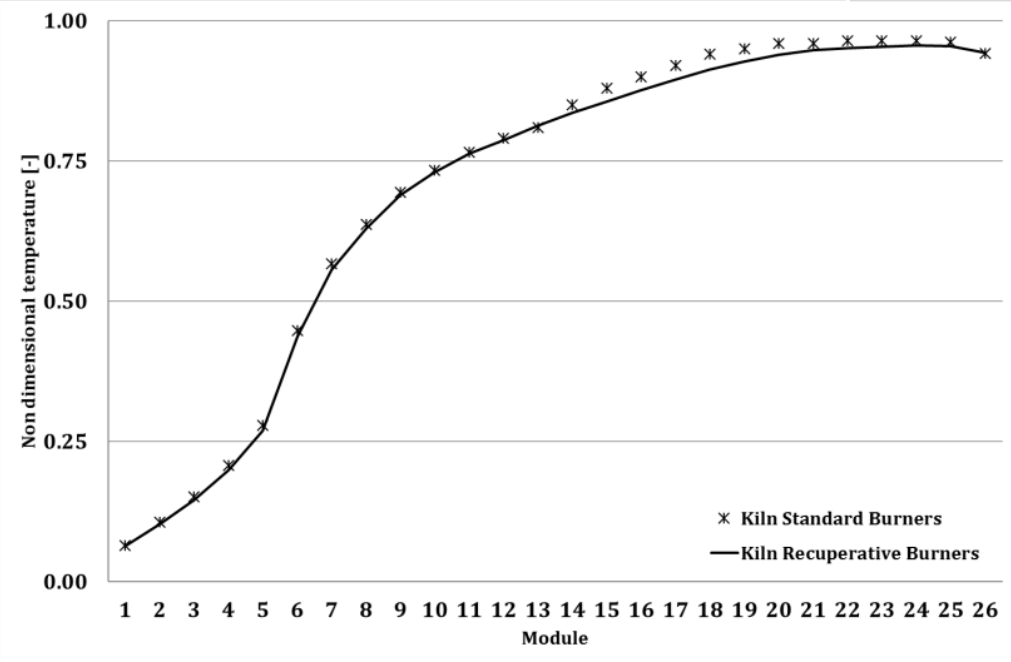

Figure 15. Comparison of the ceramic tile temperatures obtained using the standard and the heat-recovery burner

Figure 16 plots the methane consumption during the production phase for each group of burners and the results obtained using the standard and the heat-recovery burners are compared. The simulation outlines that in the modules where the new burners are employed the consumption of methane is lower, while the sections still equipped with the standard version of the burner are characterized by a larger fuel consumption. Therefore, the heat recovered from the main hot air flow to pre-heat the oxidizing air determines an advantage in the global heat transfer process. Conversely, in the initial modules, where the standard burners are employed, the consumption results slightly larger than in the old configuration, since the thermal power from the subsequent regions of the kiln is lower due to a smaller hot air flow rate and more fuel must be burned to reach the set point. Nevertheless, the balance between these two opposite behaviors result advantageous in terms of global energy efficiency of the kiln, since the total fuel consumption of the configuration equipped with the heat-recovery burners result lower by approximately the $10 \%$, see Figure 17. The fuel saving determines also a direct benefit in terms of lower carbon dioxide emissions.

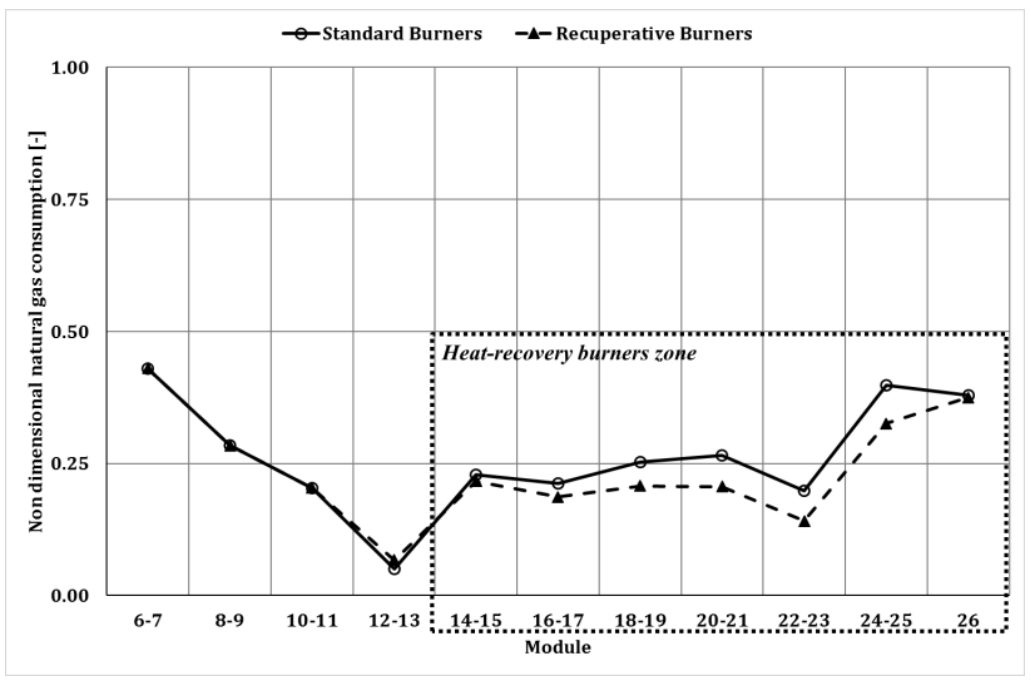

Figure 16. Comparison of the methane consumption during the production phase for each group of burners obtained using the standard and the heat-recovery burner 


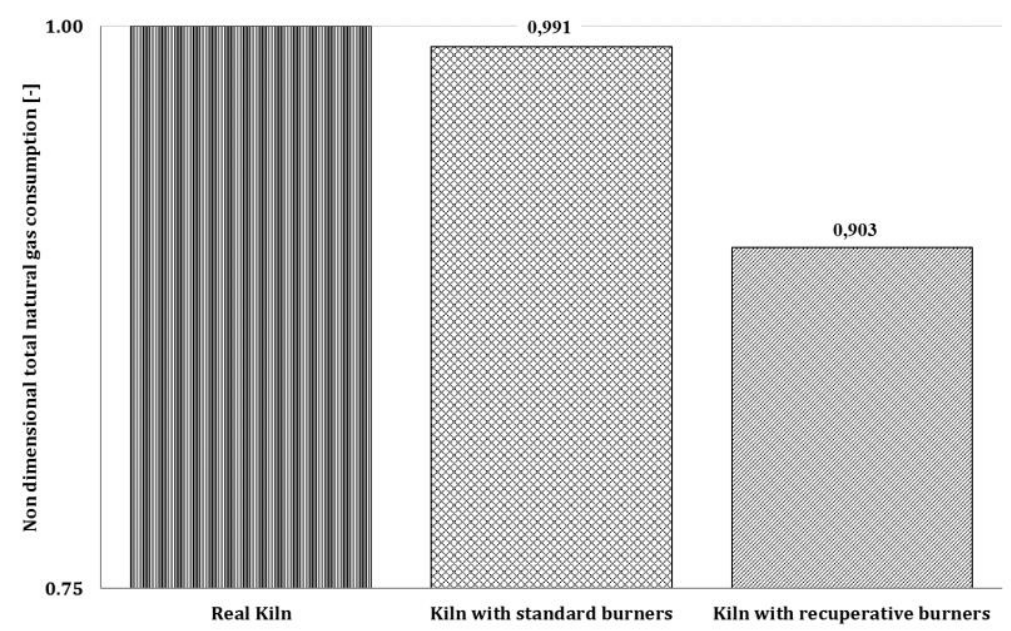

Figure 17. Measured and calculated values of the kiln fuel consumption and comparison with the fuel consumption of the configuration equipped with the heat-recovery burner

\section{CONCLUSION}

An industrial ceramic kiln has been analyzed and its thermo-fluid-dynamics behavior was investigated by means of a lumped and distributed parameter approach.

First, the numerical model of a real ceramic kiln has been constructed paying careful attention to include the complete heat transfer processes among the tiles, the hot air flow, the burner and the solid elements of the kiln. Leakages between the kiln chambers and the ambient were also accounted for as well as the control strategy of the burners was implemented into the numerical model.

The simulation results were validated by comparing the numerical prediction with the experimental measurements carried out on the real kiln under standard production operations. A good agreement between calculated values and measurements were found in terms of temperature of the hot air flow along the kiln length, the rollers' temperature and the ceramic tile temperature.

Finally, the numerical model of the kiln was employed to evaluate the effects of a new heat-recovery burner on the total fuel consumption at the same operating conditions. The lumped and distributed parameter model of the new burner was constructed and adopted to replace the standard version in the final modules of the kiln. The heat-recovery burners were then operated in order to obtained the same tile temperature and thus the total kiln fuel consumption of the old and new configurations were compared holding constant the product quality.

The simulation proved that the new heat-recovery burner enables to save up to $10 \%$ of fuel; hence, also the carbon dioxide emissions can be reduced of the same amount. 


\section{NOMENCLATURE}

\begin{tabular}{|c|c|}
\hline$\dot{m}$ & Mass Flow Rate \\
\hline$A$ & Restriction area \\
\hline$C_{q}$ & Efflux coefficient \\
\hline$C_{m}$ & Mass flow rate parameter \\
\hline$p_{0}$ & Inlet absolute pressure \\
\hline$T_{0}$ & Inlet temperature \\
\hline$k$ & Isentropic expansion exponent \\
\hline$r$ & Gas characteristic constant \\
\hline$p_{1}$ & Outlet absolute pressure \\
\hline$c_{p}$ & Specific heat \\
\hline$c_{p 0}$ & Specific heat at reference temperature \\
\hline$b_{c}$ & Constant coefficient for specific heat \\
\hline$b_{t}$ & Specific heat coefficient for temperature term \\
\hline$b_{t 2}$ & Specific heat coefficient for squared temperature term \\
\hline$\Delta T$ & Variation of the temperature \\
\hline$d h_{\text {cond }}$ & Conduction heat flow \\
\hline$\lambda$ & Thermal conductivity of the material \\
\hline$A_{\text {cond }}$ & Conduction Exchange Area \\
\hline th & Distance between the two temperature side under consideration \\
\hline$T_{1}$ & Temperature at side 1 \\
\hline$T_{2}$ & Temperature at side 2 \\
\hline$d h_{\text {conv }}$ & Convection heat flow \\
\hline $\mathrm{Nu}$ & Nussuelt number \\
\hline$c l$ & Characteristic length of the heat exchange \\
\hline$A_{\text {conv }}$ & Convection Exchange Area \\
\hline$T_{f}$ & Temperature of the fluid \\
\hline$T_{w}$ & Temperature of the wall \\
\hline$N u_{\text {free }}$ & Nussuelt number for free convection \\
\hline$N u_{\text {force }}$ & Nussuelt number for force convection \\
\hline$R a$ & Rayleigh number \\
\hline $\operatorname{Pr}$ & Prandtl number \\
\hline $\operatorname{Re}$ & Reynolds number \\
\hline$d h_{\text {rad }}$ & Radiative heat flow \\
\hline$\sigma$ & Stefan-Boltzmann constant \\
\hline$\varepsilon_{i}$ & Emitting coefficient of body $i$ \\
\hline$\varepsilon_{j}$ & Emitting coefficient of body $j$ \\
\hline$A_{i}$ & Emitting area of body $i$ \\
\hline$A_{j}$ & Emitting area of body $j$ \\
\hline$F_{i j}$ & Shape factor from which $A_{i j}$ "sees" $A_{j i}$ \\
\hline$F_{j i}$ & Shape factor from which $A_{j i}$ "sees" $A_{i j}$ \\
\hline
\end{tabular}




\section{REFERENCES}

1. Christos, A, Theocharis, T., Energy saving technologies in the European ceramic sector: a systematic review, Applied Thermal Engineering, 21, pp 1231-1249, 2001.

2. Bovea, M. D., Diaz-Albo, E., Gallardo, A., Colomer, F. J., Serrano, J., Environmental performance of ceramic tiles: Improvement proposals, Material and Design, 31, pp 35-41, 2010.

3. Peng, J., Zhao, Y., Jiao, L., Zheng, W., Zeng, L., CO2 emission calculation and reduction options in ceramic tile manufacture - The Foshan case, Energy Procedia, 16, pp 467-476, 2012.

4. Kaya, S., Mançuhan, E., Küçükada, K., Modelling and optimization of the firing zone of a tunnel kiln to predict the optimal feed locations and mass fluxes of the fuel and secondary air, Applied Energy, 86, pp 325-332, 2009.

5. Mezquita, A., Boix, J., Monfort, E., Mallol G., Energy saving in ceramic tile kilns: Cooling gas heat recovery, Applied Thermal Engineering, 65, pp 102-110, 2014.

6. Nicolau, V. deP., Dadam, A. P., Numerical and Experimental Thermal Analysis of a Tunnel Kiln used in Ceramic Production, Journal of Brazilian Society of Mechanical Science and Engineering, Vol 31, pp 297-304, 2009.

7. Mercati, S., Milani, M., Montorsi, L., Paltrinieri, F., Design of the steam generator in an energy conversion system based on the aluminum combustion with water, Applied Energy, 97, pp. 686 - 694, I.F. 1 year 5.106 - 5 year 4.456, 2012.

8. Franzoni, F., Mercati, S., Milani, M., Montorsi, L., Operating Maps of a Combined Hydrogen Production and Power Generation System Based on Aluminum Combustion with Water, International Journal of Hydrogen Energy, 36, pp. 2803 - 2816, ISSN: 0360-3199, I.F. 1 year 3.452 - 5 year 4.028, 2011.

9. Milani, M., Montorsi, L., Stefani, M., An integrated approach to energy recovery from biomass and waste: Anaerobic digestion-gasification-water treatment, Waste Management \& Research, 32 (7), pp 614-625, ISSN: 1096-3669, 2014.

10.Bottazzi, D., Farina, S., Milani, M., Montorsi, L., A numerical approach for the analysis of the coffee roasting process, Journal of Food Engineering, 112 (3), pp. 243 - 252, I.F. 1 year2.4145 year 2.805, 2012.

11.LMS Imagine.LAB. (2016) AMESet Rev 14, Reference Manual.

12.Reid, R.C. and Sherwood, T., The properties of gases and liquids. Their estimation and correlation. McGraw Hill Chemical Engineering Series, 2001.

13.Gasunie, Physical properties of natural gases, N.V. Nederlandse Gasunie, Groningen (the Netherlands), 1998.

14.Franzoni, F., Milani, M., Montorsi, L., A CFD Multidimensional Approach to Hydraulic Components Design, SAE 2007-01-4196; Published also by "SAE - 2007 Transactions Journal of Commercial Vehicles”, pp. 246 - 259, ISBN 978-0-7680-1981-0, 2007. 